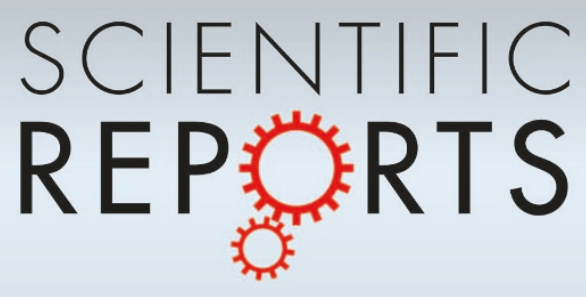

OPEN

SUBJECT AREAS:

NEMS

NANOSCALE BIOPHYSICS

Received

14 March 2014

Accepted

25 July 2014

Published

13 August 2014

Correspondence and requests for materials should be addressed to

J.T. (jłamayo@imm. cnm.csic.es)

\section{Physics of Nanomechanical Spectrometry of Viruses}

\author{
J. J. Ruz, J. Tamayo, V. Pini, P. M. Kosaka \& M. Calleja
}

Institute of Microelectronics of Madrid, CSIC. Isaac Newton 8 (PTM), Tres Cantos. E-28760 Madrid, Spain

There is an emerging need of nanotools able to quantify the mechanical properties of single biological entities. A promising approach is the measurement of the shifts of the resonant frequencies of ultrathin cantilevers induced by the adsorption of the studied biological systems. Here, we present a detailed theoretical analysis to calculate the resonance frequency shift induced by the mechanical stiffness of viral nanotubes. The model accounts for the high surface-to-volume ratio featured by single biological entities, the shape anisotropy and the interfacial adhesion. The model is applied to the case in which tobacco mosaic virus is randomly delivered to a silicon nitride cantilever. The theoretical framework opens the door to a novel paradigm for biological spectrometry as well as for measuring the Young's modulus of biological systems with minimal strains.

$\mathrm{t}$ is increasingly evident the intimate link between the mechanical properties of biological systems and its role in fundamental biological processes and disease ${ }^{1}$. This link spans from the molecular scale to the tissue scale.

For example, the elasticity of cells has become a reliable indicator of cell transformation into cancerous or metastatic cells ${ }^{2-3}$. Similarly, recent reports have demonstrated the biological relevance of the mechanical properties of viruses. Viruses are able to dynamically modulate their mechanical properties in response to external forces, so as to withstand those forces or to ease cell infection ${ }^{4}$. For instance, in the human immunodeficiency and murine leukemia viruses, the stiffness largely decreases during the maturation process, acting as a mechanical switch for the infection process ${ }^{5}$. Strikingly, a single point mutation in the capsid protein of some viruses can significantly change their elasticity ${ }^{6}$. It is therefore fundamental the development of nanotools that enable the accurate quantification of the nanomechanical properties of single biological entities with high throughput. These tools can provide new insights on how the structural conformation, biological function, and mechanical properties of biomolecules and their hierarchical assemblies are related each other. The most prominent method to measure the mechanical properties of biological entities has been so far nanoindentation with the cantilever/tip assembly of an atomic force microscope $(\mathrm{AFM})^{7}$. However, a number of challenges exist with the AFM for the quantification of the mechanical properties. Mainly, the nanoindentation curves strongly depend on the nanometer-scale geometry of the tip/sample contact, which in most of the cases cannot be controlled. Other difficulties include the contribution of the underlying substrate, the effect of adhesion, non-linear loading and the lack of accurate theoretical models.

We envisage a novel biological spectrometry technique based on the measurement of several vibration modes of ultrathin micro- and nanocantilevers for the identification of adsorbed biomolecules and biological systems by two coordinates: the mass ${ }^{8-11}$ and its stiffness ${ }^{1}$. The use of ultrathin cantilevers with thickness below $100 \mathrm{~nm}$ is justified to boost the stiffness effect. The proposed technology is feasible as ultrathin cantilevers can routinely be fabricated and methods for the delivery of biological particles one by one to the resonator in vacuum have been demonstrated $^{9-10}$. A key piece in this approach is a model that accounts for the effect of the stiffness of the biological particles on the recorded jumps in the resonant frequencies. So far, the only model that accounts for this effect assumes that the adsorbate length is much larger than its thickness and hence the adsorbate can effectively be approximated by a thin layer on a cantilever region ${ }^{12}$. The case of single biological entities, such as proteins and viruses, is significantly more challenging. These objects have arbitrary shapes more complex than a simple thin layer. Furthermore, they significantly deform when they adsorb on a surface due to the interfacial energy and their low Young's modulus. Moreover, a single biological object exhibits high surface-to-volume ratio, and thereby the stress induced by the cantilever vibration can significantly be released through the deformation of its free surface. Finally, the nanomechanical response may be anisotropic in the case of rod-like nanoobjects.

Here, we develop an analytical model for the accurate determination of the Young's modulus of single biological entities by using nanomechanical resonators that accounts for the high surface-to-volume ratio fea- 


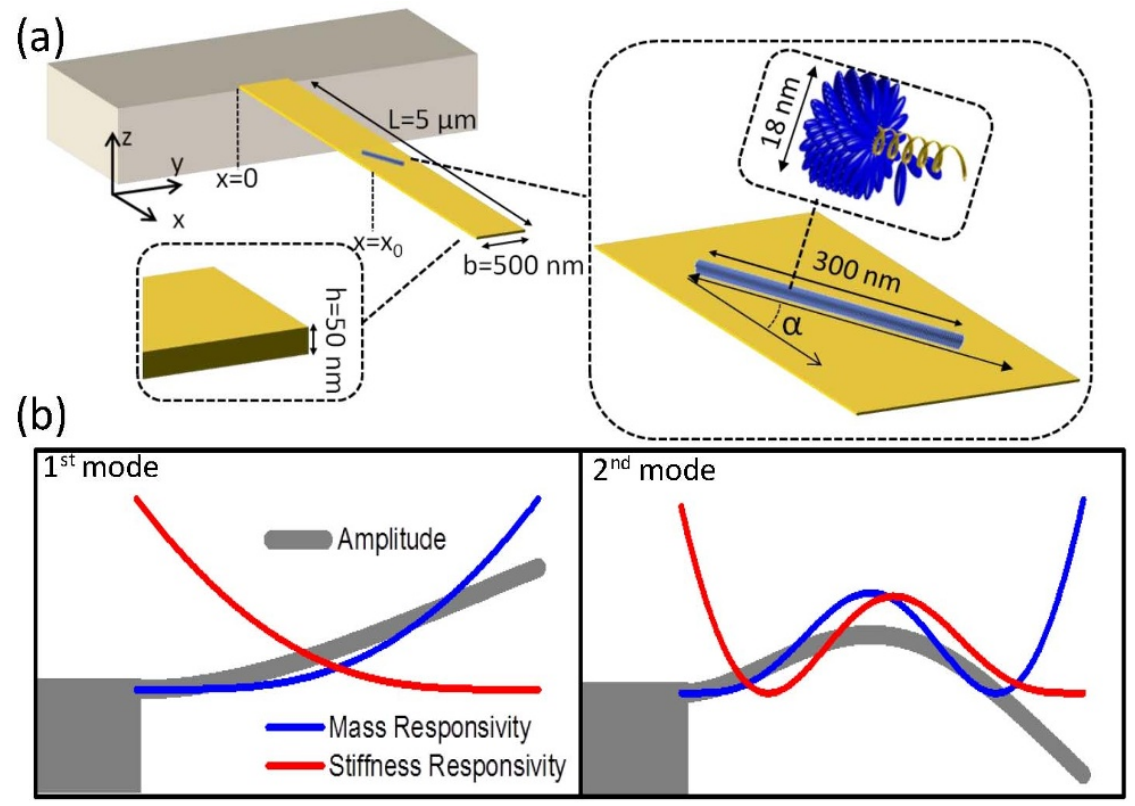

Figure 1 Nanomechanical spectrometry of viral particles. (a) Schematic of the tobacco mosaic virus (TMV) on a cantilever showing the coordinate system and dimensions used in this work. (b) Vibration mode shape, mass responsivity and stiffness responsivity of the first two vibration modes of the cantilever.

tured by single biological entities, the shape anisotropy and the interfacial adhesion. In particular, we have modeled the effect of the adsorption of tobacco mosaic virus (TMV) on a silicon nitride cantilever.

\section{Results and discussion}

System description. Silicon nitride cantilevers were chosen because they can be fabricated with very small thickness and very high yield ${ }^{13}$. However, the results presented here can be applied to any other material. The chosen values of Young's modulus, Poisson's ratio and density for the silicon nitride cantilevers are $104 \mathrm{GPa}, 0.23$ and $3187 \mathrm{Kg} / \mathrm{m}^{3}$, respectively ${ }^{13}$.The choice of TMV as biological adsorbate is justified by several reasons. First, the analysis of whole viruses by conventional mass spectrometry presents a number of technical challenges that have proven difficult to overcome $e^{14-15}$. The measurement of the Young's modulus and mass of intact viruses by nanomechanical resonators can significantly contribute to address relevant questions such as the viral variability, evolutionary changes and infective potential. Second, the TMV is an extensively studied virus by different techniques, and hence its structure, mass and mechanical properties are well-established. TMV is rod-shaped and is formed from approximately 2130 identical protein subunits wound in a $300-\mathrm{nm}$ long helix with a diameter of $18 \mathrm{~nm}^{16-17}$. A central hollow cylindrical core holds the viral genome-a 6395-nucleotide strand of RNA. TMV has a calculated molecular weight of $40.5 \mathrm{MDa}$ that provides a mass density of $880 \mathrm{~kg} / \mathrm{m}^{3}$. The axial Young's modulus of TMV has been characterized by measuring the bending rigidity and radial compression in AFM experiments ${ }^{17-18}$. The experiments indicate that the axial and radial Young's moduli are of about $6 \mathrm{GPa}$ and $1 \mathrm{GPa}$, respectively. Finally, since the TMV is rod-shaped, the adsorption effect on the resonance frequencies of the microcantilever depends on the rod orientation, which adds complexity, but it also makes the study more general and applicable to biological systems with oval and tubular shapes.

Figure 1(a) shows a schematic depiction of a TMV adsorbed on the cantilever. The cantilever is oriented along the $x$ axis with flexural displacement along the $z$ axis. The origins of the $x$ and $z$ axes are situated at the clamping and at the upper cantilever surface, respect- ively. The cantilever and TMV lengths are referred to as $L$ and $L_{a}$. The $x$-coordinate of the TMV center of mass is referred to as $x_{0}$ and the angle between the longitudinal axes of the cantilever ( $x$-axis) and the virus is referred to as $\alpha$. Our simulations indicate that the $y$-coordinate of the adsorption position plays a negligible role in the flexural resonance frequencies, and therefore the TMV center of mass is positioned at the cantilever longitudinal symmetry axis that corresponds to $y=0$. The cantilever dimensions in our simulations are: $L=5 \mu \mathrm{m}$, width $b=500 \mathrm{~nm}$ and thickness $h=50 \mathrm{~nm}$.

Figure 1(b) shows the vibration shape together with the resonance frequency responsivities to the mass and stiffness of the adsorbate for the first two vibration modes of a cantilever based on the EulerBernoulli beam theory ${ }^{1}$. The mass responsivity is negative and scales with the vibration amplitude (higher kinetic energy), whereas the stiffness responsivity is positive and scales with the vibration curvature (higher potential energy). In the first vibration mode, the region with highest amplitude is at the free end, which in turn exhibits null curvature. Conversely, the region with highest curvature is at the fixed end that in turn exhibits null amplitude. This property enables the disentanglement of the mass and stiffness effects by restricting the adsorption to the cantilever extremes ${ }^{19}$. Similarly, for the second vibration mode, the stiffness and mass effects can be uncoupled when adsorption occurs near the fixed or free end, respectively. In addition, adsorption around the vibration node induces a purely mechanical effect on the resonance frequency.

Effect of the Young's modulus of the virus. Figure 2(a) shows the numerical calculations by the finite element method (FEM) of the relative resonance frequency shifts of the first two vibration modes as a function of the adsorption position $x_{0}$ normalized to the cantilever length, $L$, and the TMV orientation angle $\alpha$. The computational details are described in the Supplementary Materials (section S1). In these simulations, we have tried to capture the most realistic shape of the TMV adsorbed on the cantilever. It is known that TMV adopts a "flattened" shape when it adsorbs on a surface as a result of the attachment of as many protein moieties as possible to the surface to minimize the surface and interfacial energies ${ }^{16}$. In figure 2(a), we have chosen a typical value for the work of adhesion of $70 \mathrm{mN} /$ $\mathrm{m}^{20-21}$. The resulting contact area and adhesion force are $7.4 \times$ 

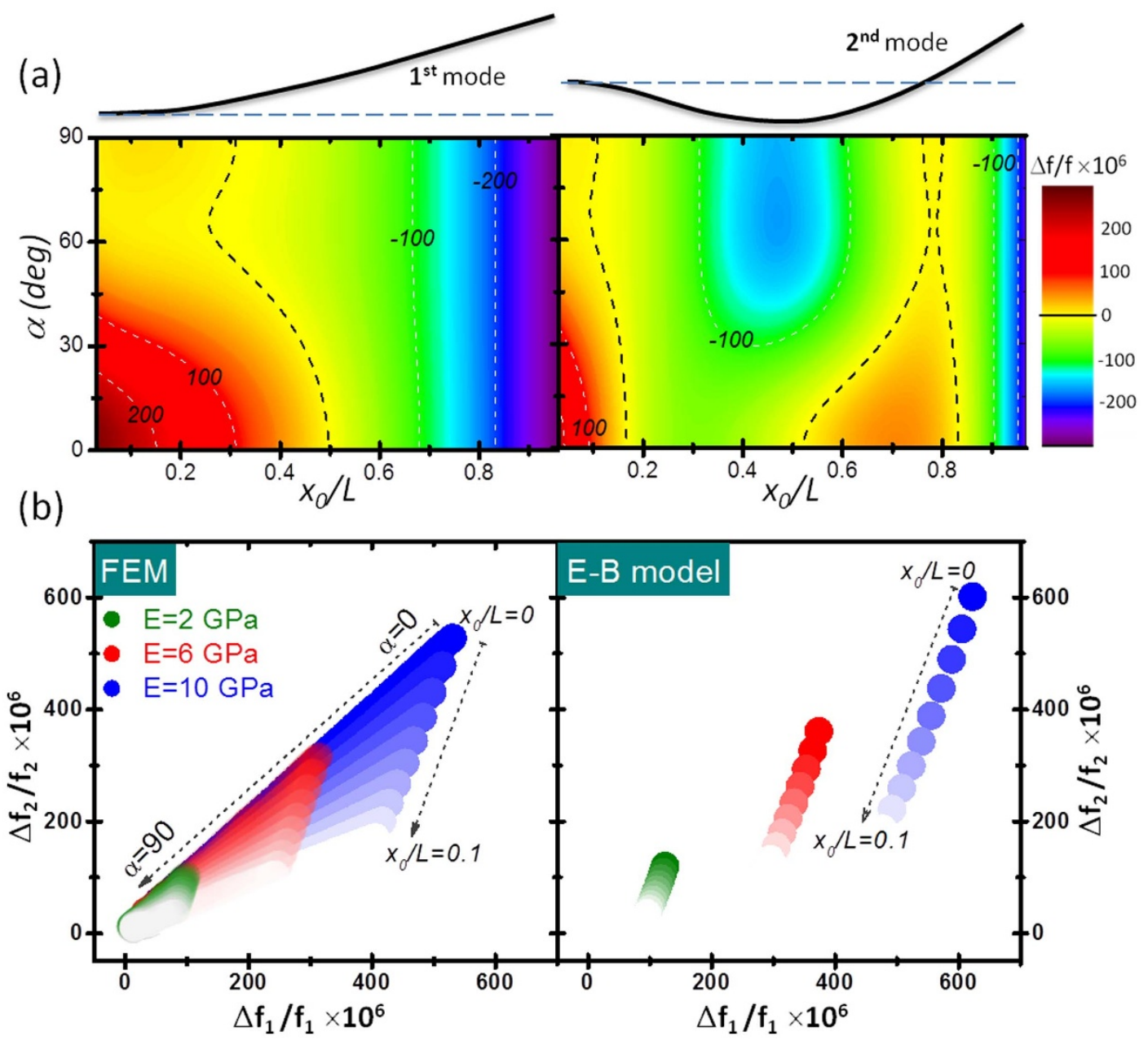

Figure 2 (a) FEM calculations of the relative resonance frequency shifts of the first two vibration modes as a function of the adsorption position $x_{0}$ normalized to the cantilever length, $L$, and TMV orientation angle $\alpha$. The black dashed contour line corresponds to zero frequency shift. (b) Adsorption position $\left(x_{0}\right)$ and TMV orientation $(\alpha)$ contours in the plane formed by axes that represent the fractional resonance frequency shift of the first two modes, $\Delta f_{1} / f_{1}$ and $\Delta f_{2} / f_{2}$ when adsorption occurs near the clamping $\left(x_{0} / L<0.1\right)$ for three values of the Young's modulus of the TMV around the nominal value. The left graph shows the FEM data and the right graph shows the analytical data calculated by the model based on the Euler-Bernoulli beam theory.

$300 \mathrm{~nm}^{2}$, and $460 \mathrm{nN}$, respectively. The role of the adhesion-induced deformation of the virus is analyzed in detail later. For the sake of understanding and simplicity, we have assumed that the TMV deformation during the cantilever vibration is mostly determined by the axial Young's modulus, $6 \mathrm{GPa}$, whereas the adhesioninduced deformation is mostly determined by the radial Young's modulus, $1 \mathrm{GPa}$. The data in Fig. 2(a) shows that the absolute value of the fractional frequency shifts induced by the TMV adsorption are $\sim 10^{-4}$, which can be measured in air and more precisely in vacuum ${ }^{22}$. The sign can be positive or negative depending on the adsorption position as described above. More interestingly, the frequency shift strongly depends on the TMV orientation, $\alpha$, when the TMV adsorbs on positions where the stiffness effect dominates, whereas it exhibits an isotropic response when the virus adsorbs on positions where the mass effect dominates. The maximum frequency shift induced by the TMV stiffness is found when the TMV is parallel to the $\mathrm{x}$ axis, whereas the minimum is found close to the perpendicular orientation.

Let us now analyze the resonance frequency response in the framework of the Euler-Bernoulli beam theory, and thereby delineate the differences between this model and the FEM data. The resonance frequency of the cantilever can be quantified by equaling the mean potential and kinetic energies per oscillation cycle, referred to as Rayleigh-Ritz method ${ }^{12}$,

$$
f_{n}^{2}=\frac{1}{4 \pi^{2}} \frac{\int_{V} \sigma_{i j} \varepsilon_{k l} \delta_{i k} \delta_{j l} d V}{\int_{V} \rho\left(u^{2}+v^{2}+w^{2}\right) d V}
$$

where $u, v$ and $w$ are the displacements in the $x, y$ and $z$ directions, $\sigma_{i j}$ and $\varepsilon_{k l}$ are the corresponding components of the stress and strain tensors, respectively, $\rho$ is the density and $\delta_{i k}$ is the Kronecker delta. Euler-Bernoulli beam theory exclusively takes into account the relevant displacements and strains generated during the cantilever vibration bending, i.e., the displacement $w$ involved in the kinetic energy term and the strain component $\varepsilon_{x x}$ involved in the potential energy, referred to as $\varepsilon_{x x}^{b}$. Both parameters are related each other by,

$$
\varepsilon_{x x}^{b}=-\left(z+\frac{h}{2}\right) \frac{d^{2} w}{d x^{2}}
$$

If we now assume that the strain in the adsorbate is just a prolongation of the strain in the cantilever (eq. (2)), equation (1) largely simplifies, and the fractional resonance frequency shift for small adsorbates can be expressed as the sum of a negative term related to the inertial effect and a positive term related to the bending stiffness effect, respectively given by (Supplementary Section S2) ${ }^{1,12}$,

$$
\left(\frac{\Delta f_{n}}{f_{n}}\right)_{m} \cong-\frac{1}{2} \psi_{n}{ }^{2}\left(\zeta_{0}\right) \frac{m_{a}}{m_{c}}
$$



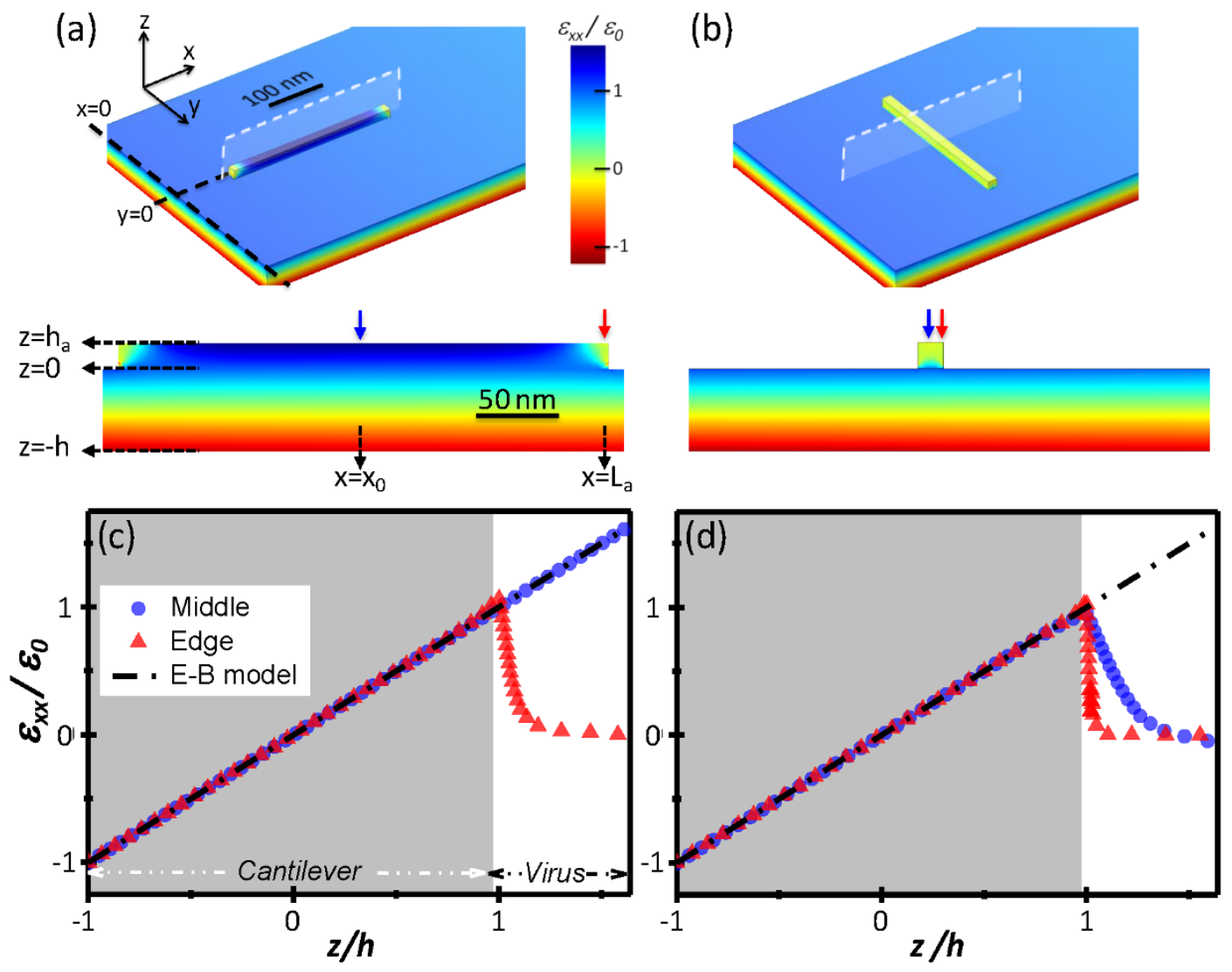

Figure 3 Strain distribution in the cantilever/TMV assembly. (a), (b). 3D and 2D colour intensity graphs of the strain component $\varepsilon_{x x}$ when the cantilever bends for the TMV oriented parallel and orthogonal to the cantilever, respectively. The 2D graphs are a slice in the $x z$ plane of the region marked in the 3D graph. (c), (d). Cross-sections of the strain $\varepsilon_{x x}$ along the blue and red arrows marked in the 2D graphs shown in (a) and (b), respectively. The grey region represents the cantilever. The model based on the Euler-Bernoulli beam theory is plotted as black dashed line. The strain is normalized by its value at the surface of the cantilever $(z=0)$ at $x=x_{0}$, referred to as $\varepsilon_{0}$.

$$
\left(\frac{\Delta f_{n}}{f_{n}}\right)_{b} \cong \frac{1}{\beta_{n}{ }^{4}} p(\eta) \frac{d^{2} \psi_{n}\left(\zeta_{0}\right)^{2}}{d \zeta^{2}} \frac{E_{a} V_{a}}{E_{c} V_{c}}
$$

where $V$ is the volume, $m$ is the mass, $E$ is the Young's modulus; the subscripts $c$ and $a$ denote the cantilever and the adsorbate, respectively; $\zeta$ is the $x$-coordinate normalized by the beam length $(L)$, thereby $\zeta_{0}=\frac{x_{0}}{L} ; \psi_{n}$ and $\beta_{n}$ are the vibration shape (eigenfunctions) and eigenvalues of the $n^{\text {th }}$ mode obtained from the solution of the Euler-Bernoulli beam equation ${ }^{1}$ (Supplementary Section S2), respectively. The eigenfunctions are normalized so that $\int_{0}^{1} \psi_{n}(\zeta)^{2} d \zeta=1$. Finally, $p$ is the ratio of the second moment of area per unit area of the adsorbate to that of the cantilever. The function $p$ is related to the shape of the adsorbate cross-section and depends on the ratio between the adsorbate and cantilever thickness referred to as $\eta$. For adsorbates with rectangular cross-section $\eta=\eta_{r e c}=\frac{h_{a}}{h}$, and $p$ is given by,

$$
p_{r e c}\left(\eta_{r e c}\right)=\frac{3}{2}+3 \eta_{r e c}+2 \eta_{r e c}^{2}
$$

Hereinafter, the manuscript will deal with the mechanical effect of the virus on the cantilever eigenfrequencies. So we assume that the virus adsorbs near the cantilever clamping where the inertial effect is negligible. Confining biological adsorption to the clamping region has previously been demonstrated for detection of DNA hybridization by fabricating arrays of silicon nitride microcantilevers with sensing gold areas alternately placed on the free and fixed cantilever ends $^{19}$. The Au areas act as sensing regions as they can be selectively bio-functionalized by means of thiol chemistry. For nanomechanical spectrometry in vacuum, we envision the use of nanostencil-based technology for the delivery of biological systems through the apertures of a thin membrane corresponding to the free and fixed end regions of the cantilever. This concept has been previously demonstrated for precisely positioning nanoparticles on cantilevers ${ }^{23}$.

Figure 2(b) shows the adsorption position $\left(x_{0}\right)$ and TMV orientation $(\alpha)$ contours in the plane formed by axes that represent the relative frequency shifts of the first two modes when adsorption occurs near the clamping $\left(\frac{x_{0}}{L}=0.1\right)$. The left graph shows the FEM data, whereas the right graph shows the data of the model based on the Euler-Bernoulli beam theory (eqns. (3) and (4)), referred to as $E-B$ model henceforth. The figure shows the contours for three values of the Young's modulus of the TMV around the nominal value. The FEM data show that the resonance frequency shifts strongly depend on the TMV orientation with respect to the cantilever, being maximal when the TMV axis is parallel to the longitudinal cantilever axis and minimal when the axes are near orthogonal. The anisotropy in the mechanical response prevents the uncoupling of the Young's modulus, adsorption position, and orientation of TMV by measuring the first two vibration modes. The determination of the stiffness of non isotropic adsorbates thus requires of the measurement of at least three vibration modes. This issue will be analyzed elsewhere. The $E-B$ model fails in the prediction of the resonance frequency responses; firstly it provides resonance frequency shifts significantly higher than the FEM values, and secondly the predicted dependence on the TMV orientation is negligible, whereas the FEM simulations show a huge dependence on $\alpha$. Thus, the resonance frequencies predicted by the 
$E-B$ model are $15-20 \%$ higher than the FEM values for $\alpha=0$, and about 12 times higher for $\alpha=90 \mathrm{deg}$.

Strain distribution in the adsorbate. To get insight into the origin of the discrepancy between the E-B model and the FEM data, we examine the strain distribution in the virus. For the sake of understanding, we approximate, in the FEM simulations, the TMV cross-section to a square with the same area than that of the TMV. Figures 3(a) and 3(b) show the distribution of the strain component $\varepsilon_{x x}$ in the TMV and near cantilever region when the cantilever is subject to flexural bending for $\alpha=0$ and $\alpha=90 \mathrm{deg}$, respectively. Figures 3(c) and 3(d) show the strain versus $z$ at the middle of the TMV $\left(x=x_{0}\right)$ and near the TMV edge, for $\alpha=0$ and $\alpha=90 \mathrm{deg}$, respectively. The strain has been normalized by its value at the top surface of the cantilever $(z=0)$ at $x=x_{0}$, referred to as $\varepsilon_{0}$. We find that the strain in the cantilever increases linearly with the coordinate $z$ following the Euler-Bernoulli beam theory (equation (2), black dashed line in Figs. 3(c) and 3(d)). For $\alpha=0$, this increase continues in most of the adsorbate with the same slope except near the edges, where the strain follows a non-linear behavior, approximating to zero as $z$ approaches to the upper corners of the TMV. For $\alpha=90$, the effect of the adsorbate edges is dominant, and the strain $\varepsilon_{x x}$ is much smaller than that predicted by equation (2).

To understand this behavior, it is useful to analyze the energy costs associated to each deformation process in the adsorbate. On one hand, the cantilever flexural bending exerts a stress on the bottom side of the adsorbate. The elastic energy associated to this process is minimized when the adsorbate deforms following the cantilever bending strain, i.e., following eq. (2). On the other hand, from equilibrium conditions, we know that the normal component of the strain at the surface of the adsorbate must be zero because the surface is not subject to stress. The elastic energy associated to this process makes that the strain tends to zero within the adsorbate. As demonstrated below, the contribution of both mechanisms to the elastic energy is parameterized by the ratio of adsorbate length in the cantilever stress direction, referred to as $\Delta \mathrm{x}$, to the adsorbate thickness. Thus for $\frac{\Delta x}{h_{a}} \gg 1$ (Figs. 3(a) and 3(c)), the most important contribution to the strain is that induced by the cantilever bending described by eq. (2). Conversely, for $\frac{\Delta x}{h_{a}} \cong 1$ (Figs. 3 (b) and 3(d)), the free surface effect of the adsorbate is dominant, and thereby the strain tends to zero with the distance to the cantilever.

Analytical model for the strains in an adsorbate with rectangular cross-section. We start by developing a theoretical model to predict the effect of the stiffness of a parallelepiped on the resonance frequencies of the cantilever. The length, width and height of the parallelepiped are $L_{a}, b_{a}$ and $h_{a}$, respectively. Later, we will adapt the model to account for the real shape adopted by the adsorbed TMV. We assume some simplifications in order to keep the equations as simple as possible without significant loss of accuracy.
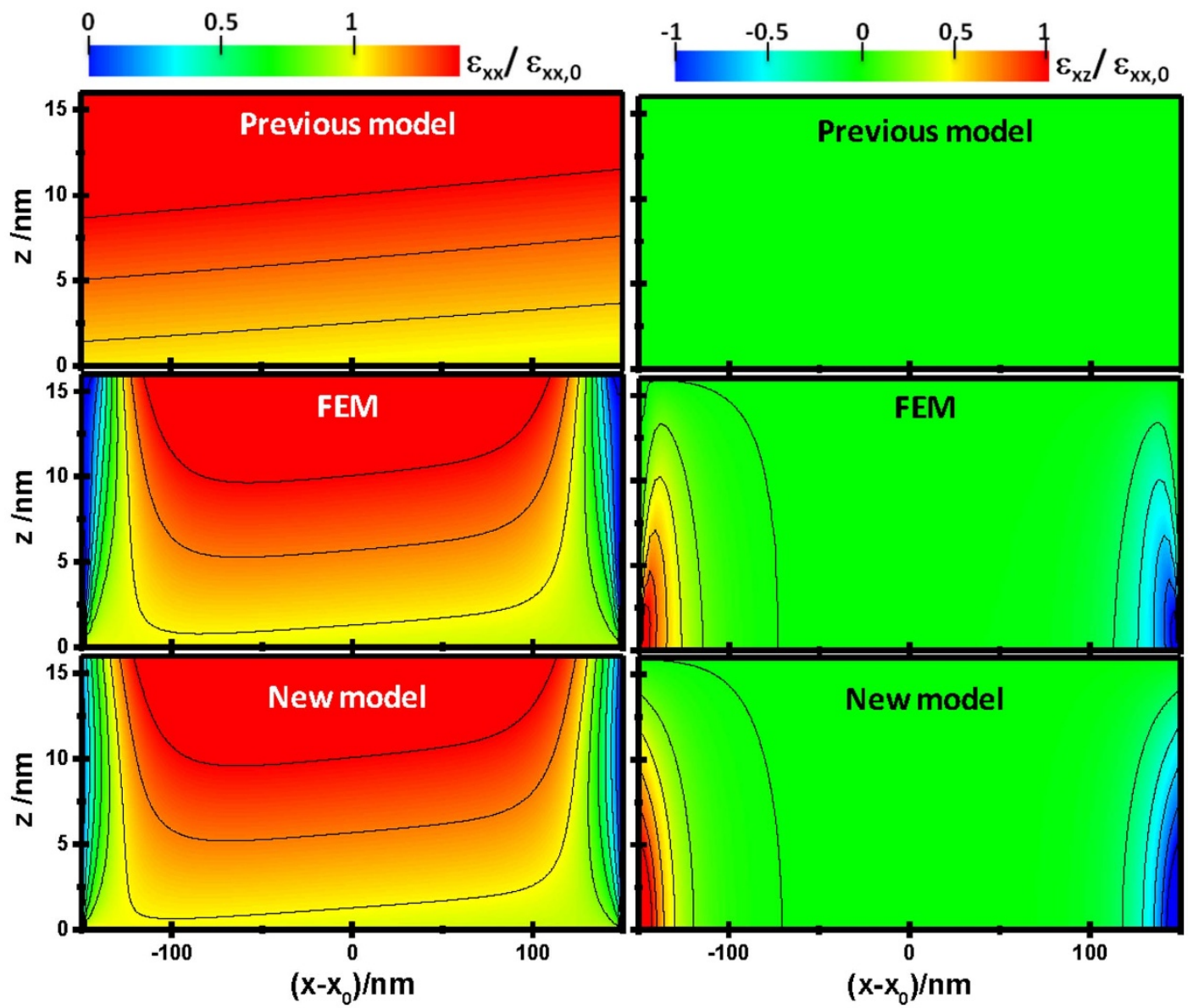

Figure $4 \mid$ Distribution of the strains $\varepsilon_{X X}$ (left) and $\varepsilon_{X z}$ (right) in the adsorbate in the plane $y=0$. The adsorbate length is that of the TMV, and the adsorbate cross-section is a square with the same area than the TMV cross-section. The adsorbate long axis is parallel to the cantilever longitudinal axis. Top graphs show the strains assumed in the $E-B$ model. Middle graphs show the strains obtained in the FEM simulations. Bottom graphs, labeled as new model, are obtained by the zero-order term in the series given by equations (9) and (10). The strains are normalized by $\varepsilon_{x x}$ at the surface of the cantilever $(z=0)$ at $x=x_{0}$, referred to as $\varepsilon_{0}$. 
First, the out-of-plane displacement, $w$, does not depend on $z$, so $\varepsilon_{z z} \cong 0$. Second, the parallelepiped is oriented parallel to the longitudinal cantilever axis, i.e., $\alpha=0$. Later, we extend the model for an arbitrary orientation. Third, we neglect the Poisson ratio of the adsorbate. The accuracy penalty of this assumption is small, and it largely simplifies the problem, especially for arbitrary orientations of the adsorbate (Supplementary Section S4). Fourth, the length of the adsorbate is much smaller than the cantilever length. Consequently, the cantilever curvature along the adsorbate can be approximated by its value at the adsorption center, $x_{0}$. Fifth, the strain is split into two summands, the strain induced by the cantilever bending, $\varepsilon_{i j}^{b}$, and the strain released through the lateral free edges of the adsorbate, $\varepsilon_{i j}^{e}$. The first term is well-described by the Euler-Bernoulli beam theory, eq. (2). The calculation of the edgeinduced strain requires solving the differential equilibrium equation in the $x$ direction $^{24}$,

$$
2 \frac{\partial^{2} u_{e}(x, z)}{\partial x^{2}}+\frac{\partial^{2} u_{e}(x, z)}{\partial z^{2}}=0
$$

where $u_{e}$ is the edge-induced displacement in the $x$ direction. We solve the differential equation (6) by the method of separation of variables, i.e., making $u_{e}(x, z)=X(x) Z(z)$. We then apply the continuity and symmetry conditions to the solution. First, the displacement solution must be continuous across the cantilever/ adsorbate interface, which implies $Z(0)=0$. Second, $X(x)$ must be an odd function about $x_{0}$ (this is strictly valid for adsorbates much shorter than the cantilever). The solution that satisfies the differential equation and the continuity and symmetry conditions is $u_{e}(x, z) \sim$ $\sinh \left[\frac{\kappa\left(x-x_{0}\right)}{\sqrt{2}}\right] \sin (\kappa z)$. By applying the strain-displacement relationships, we obtain,

$$
\begin{gathered}
\varepsilon_{x x}^{e}=\frac{\partial u_{e}}{\partial x}=C \cosh \left[\frac{\kappa\left(x-x_{0}\right)}{\sqrt{2}}\right] \sin (\kappa z) \\
\varepsilon_{x z}^{e}=\frac{1}{2} \frac{\partial u_{e}}{\partial z}=\frac{C}{\sqrt{2}} \sinh \left[\frac{\kappa\left(x-x_{0}\right)}{\sqrt{2}}\right] \cos (\kappa z)
\end{gathered}
$$

where $C$ and $\kappa$ are constants to be determined by the remaining boundary conditions. The first boundary condition dictates that the shear stress at the top free surface $\left(z=h_{a}\right)$ must be zero, which implies $\kappa=\frac{(2 n+1) \pi}{2 h_{a}}$, where $n$ can be zero and any positive integer. We form our solution as an infinite series for $n$,

$$
\begin{aligned}
& \varepsilon_{x x}^{e}=\sum_{n=0}^{\infty} C_{n} \cosh \left[\frac{(2 n+1) \pi}{2 \sqrt{2}} \chi\right] \sin \left[\frac{(2 n+1) \pi}{2} \vartheta\right] \\
& \varepsilon_{x z}^{e}=\sum_{n=0}^{\infty} \frac{C_{n}}{\sqrt{2}} \sinh \left[\frac{(2 n+1) \pi}{2 \sqrt{2}} \chi\right] \cos \left[\frac{(2 n+1) \pi}{2} \vartheta\right]
\end{aligned}
$$

where $\vartheta=\frac{z}{h_{a}}$ and $\chi=\frac{x-x_{0}}{h_{a}}$ are the longitudinal and vertical coordinates scaled to the adsorbate thickness. The second boundary condition imposes zero strain on the lateral edges of the adsorbate. This boundary condition cannot fully be reached due to the stress exerted by the cantilever at the corners of the adsorbate in contact with the cantilever. The corner pathology is circumvented by relaxing the boundary condition. Thus, we impose the minimization of the functional given by $\int_{0}^{h_{a}} d z\left(\varepsilon_{x x}^{e}+\varepsilon_{x x}^{b}\right)_{x=x_{0} \pm \frac{L_{a}}{2}}^{2}$. The functional minimization provides the coefficients $C_{n}$,

$C_{n} \cong \frac{2\left\{4(-1)^{n} \eta_{r e c}+(1+2 n) \pi\right\} h \operatorname{sech}\left[\frac{(1+2 n) \pi}{4 \sqrt{2}} \lambda_{a}\right]}{((2 n+1) \pi)^{2}} \frac{\partial^{2} w\left(x_{0} y_{0}\right)}{\partial x^{2}}$ where $\lambda_{a} \equiv \frac{L_{a}}{h_{a}}$ describes the energy balance between the cantilever bending contribution and the adsorbate free surface contribution.

We now compare the distribution of the strains predicted by our model with those derived from the $E-B$ model (eq. (2)), and with the FEM data in the plane $y=0$ (Fig. 4). For this comparison, we have chosen a parallelepiped with the same length than the TMV and with a square cross-section with area equal to the cross-sectional area of the TMV. The strains are normalized by $\varepsilon_{0},\left(\varepsilon_{x x}\right.$ at $x=x_{0}$, and $\left.z=0\right)$. The $E-B$ model neglects the shear strain and assumes that the longitudinal strain in the adsorbate is a prolongation of that in the cantilever, i.e., the strain is proportional to the coordinate $z$, with the same proportionality constant than in the cantilever (eq. (2)) (Fig. 4, top graphs). However, the FEM simulations demonstrate that first, the shear strain is significant near the adsorbate edges and, second, the longitudinal strain does not follow the bending strain of the cantilever in a region near the lateral edges with a characteristic length given by the adsorbate thickness (Fig. 4, middle graphs). In this region, the bending-induced strain tends to zero as the position approaches to the lateral edges and separates from the cantilever. In the bottom graphs of Fig. 4, we plot the bending strain (eq. (2)) plus our formulation for the strains induced by the lateral free edges. The series that describe the edgeinduced strains, eqns. (9) and (10), converges very quickly. The higher the parameter $\lambda_{a}$, the higher the rate of convergence. For $\lambda_{a}>10$, just only the first term is needed to achieve a root-mean square deviation with respect to the asymptotic limit below $3 \%$. In the case, $\lambda_{a}=1$, the series can be truncated at $n=5$. Since $\lambda_{a} \cong 18.8$ in the case discussed in Fig. 4, we only use the zero-order term in eqns. (9) and (10) (bottom graphs). The analytical formulation presented here, shows a good agreement with the numerical simulations obtained by FEM and captures the effect of the free surface of the adsorbate. More importantly, the deviation between the new model and the FEM simulations in the strain distribution significantly decreases when the elastic energy stored by the adsorbate is calculated, that in fact, it is the critical parameter to calculate the resonance frequency.

Effect of the Poisson's ratio of the cantilever. The bending cantilever strain, $\varepsilon_{x x}^{b}$, induces a transversal strain $\varepsilon_{y y}^{b}$ due to the finite Poisson's ratio that, in turn, induces edge-induced strains in the adsorbate $\varepsilon_{y y}^{e}$ and $\varepsilon_{y z}^{e}$ that are described by the same equations for $\varepsilon_{x x}^{e}$ and $\varepsilon_{x z}^{e}$ (eqns. (9) - (11)), but replacing $\chi$ by $\varsigma=\frac{y-y_{0}}{h_{a}}$, and $\frac{\partial^{2} w\left(x_{0}, y_{0}\right)}{\partial x^{2}}$ by $\frac{\partial^{2} w\left(x_{0}, y_{0}\right)}{\partial y^{2}}$. For an unrestrained plate, the transversal curvature is simply related to the longitudinal curvature through the Poisson's ratio of the plate, $\frac{\partial^{2} w}{\partial y^{2}} \approx-v \frac{\partial^{2} w}{\partial x^{2}}$. However, the transversal strain in cantilevers is constrained in a region near the clamp with a characteristic length given by the cantilever's width ${ }^{25-26}$. Since this is the relevant region to measure the mechanical properties of adsorbates, our model must account for this effect. We have recently found an analytical solution to this problem in the framework of the Stoney's equation applied to cantilevers. This model provides the following correction to our problem ${ }^{27}$,

$$
\Theta_{v} \equiv-\frac{\varepsilon_{y y}^{b}}{\varepsilon_{x x}^{b}} \approx v\left(1-e^{-\gamma(v) \frac{x}{b}}\right)
$$

where $\gamma(v)=2.28+v-0.18 v^{2}$. The transversal strain in the cantilever due to the Poisson's effect involves an increase of the strain energy in the adsorbate by a factor $\approx 1+\Theta_{v}^{2}$. 


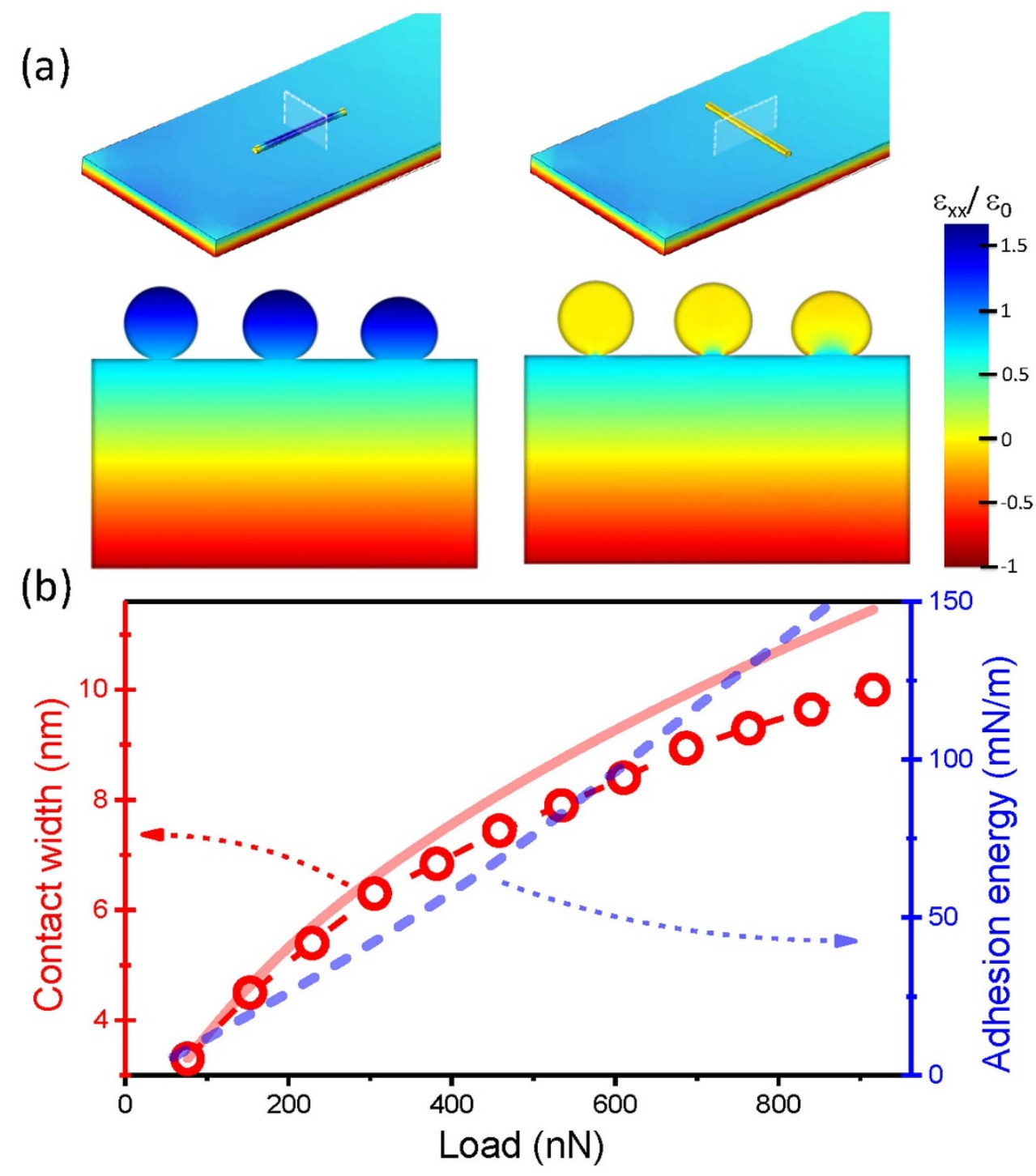

Figure $5 \mid$ (a) Distribution of the strain $\varepsilon_{x x}$ in the TMV/cantilever system for parallel $(\alpha=0)$ and transversal $(\alpha=90$ deg $)$ orientation of the TMV with respect to the cantilever. The 2D slices correspond to the planes $x=x_{0}$ and $y=0$ for $\alpha=0$ and $\alpha=90$ deg, respectively. The shown three TMV cross-sections corresponds to three loads of 75, 460 and $920 \mathrm{nN}$ that give a contact width of 3.2, 7.4 and $10 \mathrm{~nm}$, respectively. (b) Width of the TMV/cantilever contact as a function of the applied load (red symbols). The Hertz model is also represented (red solid line). In the right axis, the resulting adhesion energy derived by the Barquin's model for adhesive contacts between compliant cylinders and a flat surface is plotted (blue dashed line).

Resonance frequency shift induced by the adsorption of a parallelepiped parallel to the cantilever. Now we apply the Rayleigh-Ritz method (eq. (1)) to calculate the stiffness contribution to the resonance frequency shift induced by the adsorbed parallelepiped. The total strain energy is the sum of the contributions arising from the cantilever bending and the adsorbate edges, respectively given by,

$U_{b}=\frac{E_{a}}{2} \int_{V_{a}}\left\{\varepsilon_{x x}^{b 2}+\varepsilon_{y y}^{b 2}\right\} d V=\frac{1}{12}\left(1+\Theta_{v}{ }^{2}\right) p_{r e c}\left(\eta_{r e c}\right) E_{a} V_{a} h^{2}\left(\frac{d^{2} w\left(x_{0}\right)}{d x^{2}}\right)^{2}(13)$

$$
U_{e}=\frac{1}{2} E_{a} \int_{V_{a}}\left\{\varepsilon_{x x}^{e}{ }^{2}+\varepsilon_{y y}^{e}{ }^{2}+2\left(\varepsilon_{x z}^{e}{ }^{2}+\varepsilon_{y z}^{e}{ }^{2}\right)\right\} d v
$$

The fractional resonance frequency shift due to the stiffness effect is then given by,

$$
\left(\frac{\Delta f_{n}}{f_{n}}\right)_{s}=\left(\frac{\Delta f_{n}}{f_{n}}\right)_{b}-\left(\frac{\Delta f_{n}}{f_{n}}\right)_{e} \cong \frac{1}{2} \frac{U_{b}}{U_{c}}+\frac{1}{2} \frac{U_{e}}{U_{c}}
$$

where $U_{c}=\frac{E_{c} \beta_{n}{ }^{4} b h^{3}}{24 L^{3}} A_{n}{ }^{2}$ is the potential energy of the cantilever and $A_{n}$ is the vibration amplitude. Notice that in our notation, the negative sign of the resonance frequency shift due to edge effects is used because the adsorbate becomes "softer" as a consequence of the stress released through the free edges. By substituting the strain expressions (eqns. (9) - (11)) into equation (14), we obtain a complex solution for the resonance frequency shift in terms of infinite series. By adopting some approximations, these series converge to simple analytical equations (Supplementary Materials, Section S2) that, as demonstrated below, are highly accurate to describe our problem. We therefore obtain the following equations for the fractional frequency shift,

$$
\left(\frac{\Delta f_{n}}{f_{n}}\right)_{b} \cong\left(1+\Theta_{v}^{2}\right) P_{r e c}\left(\eta_{r e c}\right) K
$$

$$
\begin{aligned}
\left(\frac{\Delta f_{n}}{f_{n}}\right)_{e} & \cong B\left(\eta_{r e c}\right)\left\{\frac{1}{\lambda_{a}} \tanh \left[\frac{p_{r e c}\left(\eta_{r e c}\right)}{B\left(\eta_{r e c}\right)} \lambda_{a}\right]+\frac{\Theta_{v}{ }^{2}}{\mu_{a}} \tanh \left[\frac{p_{r e c}\left(\eta_{r e c}\right)}{B\left(\eta_{r e c}\right)} \mu_{a}\right]\right\} K_{(17} \\
& \equiv\left(\Omega_{\mathrm{e}}\left(\eta_{r e c}, \lambda_{a}\right)+\Theta_{v}{ }^{2} \Omega_{\mathrm{e}}\left(\eta_{r e c}, \mu_{a}\right)\right) K
\end{aligned}
$$

where $\mu_{a}=\frac{b_{a}}{h_{a}}$ and, 


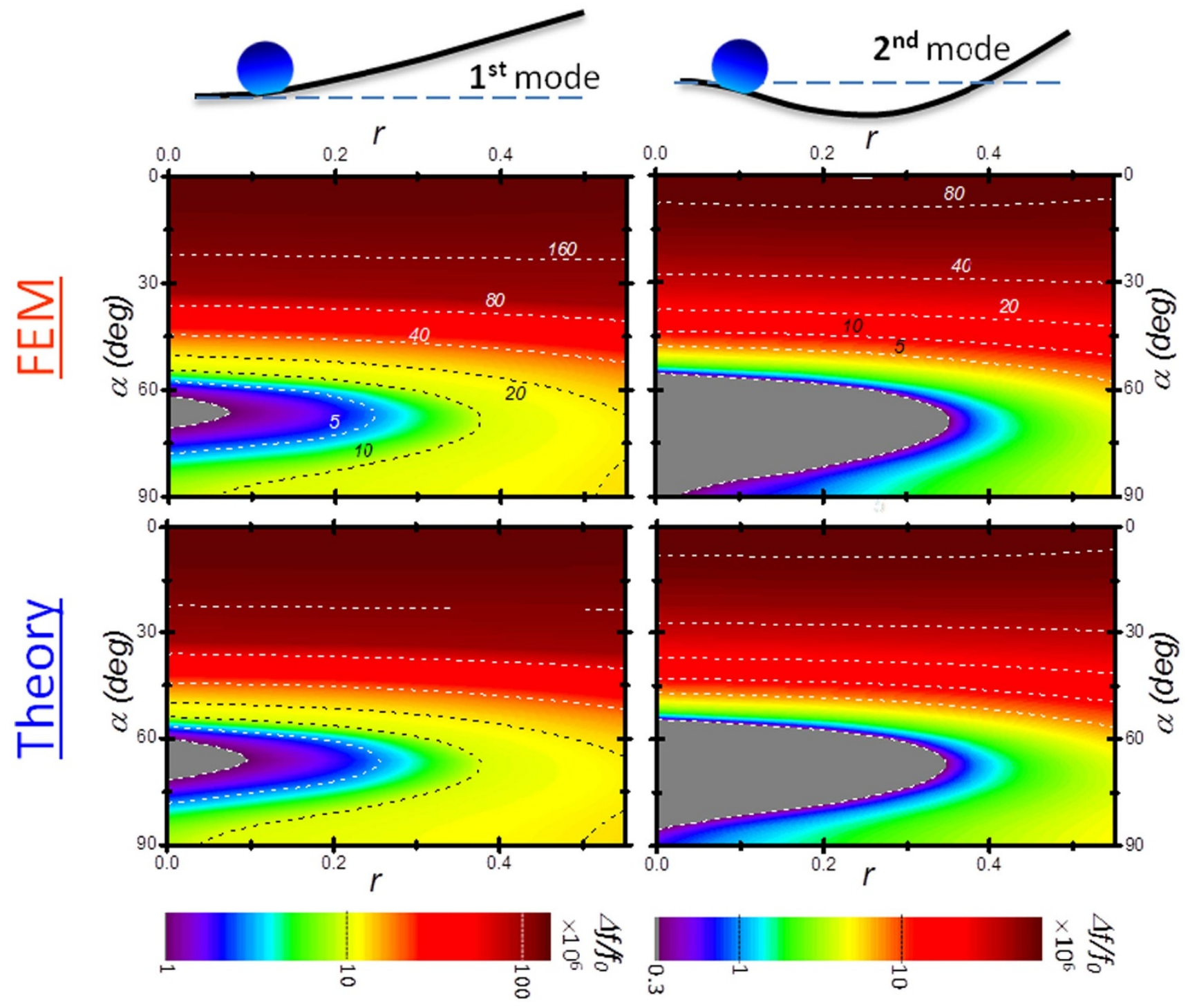

Figure 6 Comparison between the FEM simulations and our theoretical model of the resonance frequency shift induced by the adsorption of a TMV particle near the clamping, at $\frac{x_{0}}{L}=\mathbf{0 . 1}$. The graphs show the fractional frequency shifts of the first two vibration modes as a function of the TMV orientation angle, $\alpha$, and of the ratio of the TMV/cantilever contact width to the TMV diameter that accounts for the virus/cantilever adhesion. The colour intensity that represents the fractional frequency shift in ppm is log scaled. The grey regions correspond with negligible stiffness-induced resonance frequency shift, in which the inertial effect is higher and induces negative frequency shift. Notice, however, that the highest negative frequency shift is below $2 \mathrm{ppm}$. A few contour lines are plotted for ease comparison between the FEM and theory results.

$$
B\left(\eta_{r e c}\right)=\frac{3}{\sqrt{2}}\left(\frac{2}{\pi}\right)^{5}\left(\pi+4 \eta_{r e c}\right)^{2}
$$

and $K=\frac{1}{\beta_{n}{ }^{4}} \frac{d^{2} \psi_{n}\left(\zeta_{0}\right)^{2}}{d \zeta^{2}} \frac{E_{a} V_{a}}{E_{c} V_{c}}$, is the effective adsorbate mechanical stiffness relative to the cantilever.

Effect of the parallelepiped orientation. For an arbitrary orientation of the adsorbate, the problem gets more complicated, as the cantilever bending induced stress in the adsorbate is released through all the lateral edges. To solve this problem, it is useful to change from the cantilever coordinate system to the adsorbate coordinate system, i.e., the $x$ and $y$ axes are the longitudinal and transversal axes of the parallelepiped, respectively. Then, a procedure similar to that used for the case $\alpha=0$ is followed to derive the strain tensor in the adsorbate and calculate the resonance frequencies. The derivation is described in the Supplementary Materials (Section S3). Here, we provide the final simple analytical equations,

$$
\frac{\left(\frac{\Delta f_{n}}{f_{n}}\right)_{e}}{K} \cong\left(\Omega_{\mathrm{e}}\left(\eta_{r e c}, \lambda_{a}\right)+\Theta_{v}{ }^{2} \Omega_{\mathrm{e}}\left(\eta_{r e c}, \mu_{a}\right)\right) \cos ^{4} \alpha+
$$

$$
\left(\Omega_{\mathrm{e}}\left(\eta_{r e c}, \mu_{a}\right)+\Theta_{v}{ }^{2} \Omega_{\mathrm{e}}\left(\eta_{r e c}, \lambda_{a}\right)\right) \sin ^{4} \alpha+X_{e}\left(\eta_{r e c}, \mu_{a}, \lambda_{a}\right) \sin ^{2} 2 \alpha
$$

where

$$
\begin{aligned}
X_{e}\left(\eta_{r e c}, \mu_{a}, \lambda_{a}\right)= & \frac{\left(1+\Theta_{v}\right)^{2}}{2 \sqrt{2} \mu_{a}} B\left(\eta_{r e c}\right) \tanh \left(\frac{\pi}{4} \mu_{a}\right)- \\
& \frac{\Theta_{v}}{2}\left\{\Omega_{\mathrm{e}}\left(\eta_{r e c}, \lambda_{a}\right)+\Omega_{\mathrm{e}}\left(\eta_{r e c}, \mu_{a}\right)\right\}
\end{aligned}
$$

It is noteworthy to notice from the above equations that the anisotropy in the resonance frequency response to the stiffness of the adsorbate arises from the effect of the free surface, whereas the bending strain exhibits negligible dependence on the adsorbate orientation.

Extension of the theory to the adhesive contact of a tubular nanostructure and a cantilever. The developed model for adsorbates that 
are rectangular in cross-section is used as starting point for obtaining a formulation for viral nanotubes such as the TMV. This goal poses a formidable challenge because, first, the undeformed cross-section of the virus is circular, which implies that the stress can be released through all the free surface of the adsorbate; and second, the TMV structure deforms due to the adhesion interactions with the cantilever. In order to simulate the virus deformation, the FEM calculations where performed in two steps, first the virus was virtually deposited on an infinitely rigid support and was subject to a uniform normal force that mimics the adhesion force and second, the deformed shape was used for the calculation of the eigenfrequencies of the cantilever. The assumption of infinitely rigid support is justified as the cantilever Young's modulus is more than two orders of magnitude higher than that of the virus.

Figure 5(a) shows the bending strain distribution in the deformed TMV for $\alpha=0$ and $\alpha=90$ deg and for three different loads of 75, 460 and $920 \mathrm{nN}$, that give a contact width, referred to as $2 a$, of 3.2, 7.4 and $10 \mathrm{~nm}$, respectively. The contact zone between the virus and the cantilever is a strip of length, $L_{a}$ and width $2 a$. When the virus is oriented parallel to the cantilever, the cantilever bending stress is efficiently transmitted to most of the virus volume with little dependence on the contact area. Only in a region near the virus ends with length of the order the virus diameter, the strain decays to zero. When the virus is transversally oriented, the stress exerted by the cantilever along the contact width is significantly screened by the free surface of the virus. In this case, the contact width plays a critical role, and thereby the amount of bending strain within the adsorbate scales up with the contact area. In figure 5(b), we plot the contact width, $2 a$, as a function of the applied load (symbols). We compare the numerical data with the benchmark Hertz model for the contact between a cylinder and a solid flat surface ${ }^{28}$ (solid line). The Hertz model fits the FEM data satisfactorily for low loads, in which the ratio between the contact width and the virus radius is below $20 \%$. For higher loads, the Hertz model overestimates the numerical data. The deviation quadratically increases with the load. The discrepancy arises from the fact that Hertz theory assumes small deformations, and hence the geometric nonlinearities that emerge in the deformation of compliant cylinders are not considered. We relate the contact width to the adhesion energy by applying the Barquins's model that describes the adhesive contact of a compliant cylinder on a flat surface ${ }^{29-30}$ (dashed line). The adhesion energy in our simulation ranges from 5 to $150 \mathrm{mN} / \mathrm{m}$ that are the typical values found when the interaction between the virus and the cantilever is dominated by van der Waals forces $^{21}$.

In order to derive the effect of the TMV stiffness on the cantilever resonance frequency, we start with the formulation obtained for the adsorbate with rectangular cross-section, eqns. (16) - (20). Since our structure is a cylinder, we redefine the dimensionless aspect ratios of the adsorbate that characterize our problem in eq. (19). First, the virus thickness is parameterized by its diameter, $2 R$, so $\lambda_{a} \equiv \frac{L_{a}}{2 R}$ and $\eta_{\text {rec }}$ is substituted by $\eta_{c i r}=\frac{2 R}{h}$. Second, the virus deformation is accounted by the parameter $r \equiv \frac{a}{R}$. Finally, when the adsorbate is transversally oriented with respect to the cantilever, the strain develops in a region of width that is of the order of the contact width (see left graph in Fig. 5(a)). Thereby, the parameter $\mu_{a}$ is defined as $\mu_{a} \equiv r$. We start providing a solution to the resonance frequency shift induced by the cantilever bending using the functional form found for the case of the adsorbate with rectangular cross-section (eq.(16)),

$$
\begin{aligned}
\frac{\left(\frac{\Delta f_{n}}{f_{n}}\right)_{b}}{K} \cong & \left(1+\Theta_{v}^{2}\right) P_{T M V}\left(\eta_{c i r}, r\right)=\left(1+\Theta_{v}{ }^{2}\right) \\
& \left\{p_{c i r}\left(\eta_{c i r}\right)-\Delta p_{d e f}\left(\eta_{c i r}, r\right)\right\}
\end{aligned}
$$

where $p_{T M V}\left(\eta_{\text {cir }}, r\right)$ is the ratio of the second moment of area per unit area of the TMV to that of the cantilever that depends on the cross-section shape and it is described by two contributions, $p_{\text {cir }}\left(\eta_{\text {cir }}\right)=\frac{3}{8}\left(4+8 \eta_{\text {cir }}+5 \eta_{\text {cir }}^{2}\right)$, related to circular cross-section of the undeformed virus, and $\Delta p_{\text {def }}$ that accounts for the reduction of the second moment of area due to the virus deformation upon contact. The reduction of the second moment of area arises from the fact that the virus deforms adopting a "flattened" shape, and thereby the center of mass of the virus approaches to the cantilever neutral axis. The correction term due to deformation is obtained by fitting the second moment of area obtained by FEM with a second order polynomial in $\eta_{\text {cir }}$ and $r$,

$$
\Delta p_{\text {def }} \cong r\left(0.67+0.89 \eta_{\text {cir }}\right) \eta_{c i r}+r^{2}\left(0.82+0.65 \eta_{\text {cir }}\right) \eta_{c i r}
$$

The derivation of the resonance frequency shift induced by the effect of the TMV free surface starts with the functional form obtained for the adsorbate with rectangular cross-section but modified with the dimensionless parameter that characterize the TMV described above,

$$
\begin{array}{r}
\frac{\left(\frac{\Delta f_{n}}{f_{n}}\right)_{e} \cong}{K} \cong\left(\Omega_{x}\left(\eta_{c i r}, \lambda_{a}, r\right)+\Theta_{v}{ }^{2} \Omega_{y}\left(\eta_{c i r}, \mu_{a}, r\right)\right) \cos ^{4} \alpha+ \\
\left(\Omega_{y}\left(\eta_{c i r}, \lambda_{a}, r\right)+\Theta_{v}{ }^{2} \Omega_{x}\left(\eta_{c i r}, \mu_{a}, r\right)\right) \sin ^{4} \alpha+ \\
\Omega_{x y}\left(\eta_{c i r}, \lambda_{a}, r\right) \sin ^{2} 2 \alpha \\
\Omega_{x}\left(\eta_{c i r}, \lambda_{a}, r\right)=\frac{B_{x}\left(\eta_{c i r}, r\right)}{\lambda_{a}} \tanh \left[\frac{p_{T M V}\left(\eta_{c i r}, r\right) \lambda_{a}}{B_{x}\left(\eta_{c i r}, r\right)}\right] \\
\Omega_{y}\left(\eta_{c i r}, r\right)=\frac{B_{y}\left(\eta_{c i r}, r\right)}{r} \tanh \left[\frac{p_{T M V}\left(\eta_{c i r}, r\right) r}{B_{y}\left(\eta_{c i r}, r\right)}\right] \\
\Omega_{x y}\left(\eta_{c i r}, \lambda_{a}, r\right)= \\
\frac{\left(1+\Theta_{v}\right)^{2}}{2 \sqrt{2} r} B_{x y}\left(\eta_{c i r}, r\right) \tanh \left(\frac{\pi}{4} r\right)- \\
\frac{\Theta_{v}}{2}\left\{\Omega_{x}\left(\eta_{c i r}, \lambda_{a}, r\right)+\Omega_{y}\left(\eta_{c i r}, r\right)\right\}
\end{array}
$$

Notice that the function $B\left(\eta_{r e c}\right)$ obtained for the case of the parallelepiped is here split into three different functions for each angular coefficient, $B_{x}\left(\eta_{c i r}, r\right), B_{y}\left(\eta_{c i r}, r\right)$ and $B_{x y}\left(\eta_{c i r}, r\right)$, respectively. This assumption arises from the higher complexity of the strain behavior in a deformed cylinder in comparison with that in a parallelepiped. The edge-induced strain problem for a parallelepiped is the same for transversal and longitudinal orientations with respect to the cantilever, as the cross-sections in both orientations are rectangular. In the case of a cylinder parallel to the cantilever, the edge-induced strain problem is similar to that of parallelepiped as the cylinder can be divided in longitudinal rectangular cross-sections. When the cylinder is oriented perpendicularly to the cantilever, the problem is radically different as the bending strain can be released in all the radial directions through the circular-like periphery of the virus. We express the functions $B_{x}, B_{y}$ and $B_{x y}$ as second-order polynomials in $\eta_{c i r}$ and $r$. The polynomial coefficients are evaluated by performing a non-linear least-squares fit with the FEM data,

$$
\begin{gathered}
B_{x}\left(\eta_{c i r}, r\right)=4.59+11.36 \eta_{c i r}+5 \eta_{c i r}{ }^{2}- \\
\left(3.76+20.69 \eta_{c i r}-3.96 \eta_{c i r}{ }^{2}\right) r+ \\
\left(18.62 \eta_{c i r}-18.09 \eta_{c i r}{ }^{2}\right) r^{2} \\
B_{y}\left(\eta_{c i r}, r\right)=B_{y}\left(\eta_{c i r}\right)=1.72+4.05 \eta_{c i r}+4.71 \eta_{c i r}^{2}
\end{gathered}
$$




$$
\begin{aligned}
B_{x y}\left(\eta_{c i r}, r\right)= & 2.55+6.19 \eta_{c i r}+2.04 \eta_{c i r}{ }^{2}+ \\
& \left(0.88-5.18 \eta_{c i r}+4.7{\eta_{c i r}}^{2}\right) r- \\
& \left(1.87-3.28 \eta_{c i r}+9.87 \eta_{c i r}{ }^{2}\right) r^{2}
\end{aligned}
$$

In figure 6, we plot the fractional frequency shifts of the first two vibration modes obtained by FEM simulations and our theoretical model when a TMV is adsorbed near the clamp, $\frac{x_{0}}{L}=0.1$ as a function of the relative contact width $(r)$ and the TMV orientation $(\alpha)$. The plotted data demonstrate that the proposed simple analytical model not only captures the physics of the problem, but also remarkably fits the FEM simulations with high accuracy. In fact, the mean deviation between the analytical formulation and the FEM results is below $4 \%$. For both vibration modes, the higher resonance frequency shifts are achieved when the TMV's orientation approaches the parallel configuration with the cantilever. For small values of $\alpha$, the effect of the interfacial energy between the TMV and the cantilever is very small. However, this is not the case for values of $\alpha>60 \mathrm{deg}$, in which the resonance frequency shift significantly increases with the contact's width. We find a critical angle that corresponds with null stiffness-induced resonance frequency shift for null contact's width. At this critical angle, we find the highest dependence of the resonance frequency shift on the contact's width. In Fig. 6 , the critical angle is $\alpha \approx 70 \mathrm{deg}$, which significantly differs from $90 \mathrm{deg}$, the value that one may intuitively think at which the mechanical coupling between the cantilever and the virus is minimum. This intuition is correct when the Poisson ratio of the cantilever is zero, as the cantilever only induces strain in the $\mathrm{x}$-direction during the flexural vibration, and if the TMVs is transversally oriented, the amount of stress transmitted to the virus scales up with the contact's width as observed in Fig. 5, being zero for null adhesion. However, if the Poisson's ratio is not zero for $\alpha \approx 90 \mathrm{deg}$, even for null adhesion, the cantilever transversal strain due to the Poisson's effect induces stress along the transversal contact line, and hence the cantilever experiences 'mechanical impedance'. This effect shifts the critical angle to lower angles, in which the vibration and corresponding Poisson effect induced stresses cancel out each other within the virus.

\section{Conclusions}

Here, we have presented a detailed theoretical analysis to calculate the resonance frequency shift in microcantilevers induced by the mechanical stiffness of adsorbed viral nanotubes. The model accounts for the high surface-to-volume ratio featured by single biological entities that significantly 'screens' the elastic energy within the adsorbate, the anisotropy in the response due to the rod-like shape and the interfacial adhesion that influences on the mechanical coupling between the cantilever and the biological particle. The developed theoretical framework provides simple analytical equations for complex adsorbate-nanocantilever scenarios that are highly accurate when compared with finite element simulations. The model has been applied to the case in which tobacco mosaic virus is randomly delivered to a silicon nitride cantilever. However, the theory can be easily adapted to nanomechanical resonators with different geometries (e.g., doubly clamped beams and drums), and biological entities with different shapes. The theoretical framework opens the door to a novel paradigm for biological spectrometry as well as for measuring the Young's modulus of biological systems with minimal strains. We envision that the mass, position and orientation-dependent stiffness can be obtained by tracking the frequency of multiple vibration modes and solving the inverse problem ${ }^{8,10-11}$. In addition, the implementation of imaging techniques that can approximately resolve the position of the adsorbate on the cantilever can simplify the problem and reduce the error in the calculations $s^{31-32}$.
1. Tamayo, J., Kosaka, P. M., Ruz, J. J., San Paulo, Á. \& Calleja, M. Biosensors based on nanomechanical systems. Chem. Soc. Rev. 42, 1287-1311 (2013).

2. Wirtz, D., Konstantopoulos, K. \& Searson, P. C. The physics of cancer: the role of physical interactions and mechanical forces in metastasis. Nat. Rev. Cancer 11, 512-522 (2011).

3. Plodinec, M. et al. The nanomechanical signature of breast cancer. Nat. Nanotechnol. 7, 757-765 (2012).

4. Mateu, M. G. Mechanical properties of viruses analyzed by atomic force microscopy: A virological perspective. Virus Res. 168, 1-22 (2012).

5. Kol, N. et al. A stiffness switch in human immunodeficiency virus. Biophys. J. 92, 1777-1783 (2007).

6. Michel, J. et al. Nanoindentation studies of full and empty viral capsids and the effects of capsid protein mutations on elasticity and strength. P. Natl. Acad. Sci. 103, 6184-6189 (2006).

7. Kurland, N. E., Drira, Z. \& Yadavalli, V. K. Measurement of nanomechanical properties of biomolecules using atomic force microscopy. Micron 43, 116-128 (2012).

8. Tamayo, J. et al. Imaging the surface stress and vibration modes of a microcantilever by laser beam deflection microscopy. Nanotechnology 23, 315501 (2012).

9. Naik, A., Hanay, M., Hiebert, W., Feng, X. \& Roukes, M. Towards single-molecule nanomechanical mass spectrometry. Nat. Nanotechnol. 4, 445-450 (2009).

10. Hanay, M. et al. Single-protein nanomechanical mass spectrometry in real time. Nat. Nanotechnol. 7, 602-608 (2012).

11. Dohn, S., Sandberg, R., Svendsen, W. \& Boisen, A. Enhanced functionality of cantilever based mass sensors using higher modes. Appl. Phys. Lett. 86, 233501 (2005).

12. Tamayo, J., Ramos, D., Mertens, J. \& Calleja, M. Effect of the adsorbate stiffness on the resonance response of microcantilever sensors. Appl. Phys. Lett. 89, 224104224104-224103 (2006).

13. Pini, V. et al. Shedding light on axial stress effect on resonance frequencies of nanocantilevers. ACS Nano 5, 4269-4275 (2011).

14. Bothner, B. \& Siuzdak, G. Electrospray ionization of a whole virus: analyzing mass, structure, and viability. ChemBioChem 5, 258-260 (2004).

15. Fuerstenau, S. D. et al. Mass spectrometry of an intact virus. Angew. Chem. Int. Edit. 40, 541-544 (2001).

16. Knez, M. et al. Binding the tobacco mosaic virus to inorganic surfaces. Langmuir 20, 441-447 (2004).

17. Schmatulla, A., Maghelli, N. \& Marti, O. Micromechanical properties of tobacco mosaic viruses. J. Microsc. 225, 264-268 (2007).

18. Zhao, Y., Ge, Z. \& Fang, J. Elastic modulus of viral nanotubes. Phys. Rev. E 78, 031914 (2008).

19. Ramos, D. et al. Arrays of dual nanomechanical resonators for selective biological detection. Anal. Chem. 81, 2274-2279 (2009).

20. Bhushan, B. Modern Tribology Handbook, Two Volume Set. (CRC press, 2010).

21. Israelachvili, J. N. Intermolecular and surface forces: revised third edition. (Academic press, 2011).

22. Cleland, A. \& Roukes, M. Noise processes in nanomechanical resonators. J. Appl. Phys. 92, 2758-2769 (2002).

23. Engstrom, D. S. et al. High throughput nanofabrication of silicon nanowire and carbon nanotube tips on afm probes by stencil-deposited catalysts. Nano Lett. 11, 1568-1574 (2011).

24. Timoshenko, S., Woinowsky-Krieger, S. \& Woinowsky, S. Theory of plates and shells. Vol. 2 (McGraw-hill New York, 1959).

25. Karabalin, R. B., Villanueva, L. G., Matheny, M. H., Sader, J. E. \& Roukes, M. L. Stress-Induced variations in the stiffness of micro-and nanocantilever beams. Phys. Rev. Lett. 108, 236101 (2012).

26. Lachut, M. J. \& Sader, J. E. Effect of surface stress on the stiffness of thin elastic plates and beams. Phys. Rev. B 85, 085440 (2012).

27. Tamayo, J., Ruz, J. J., Pini, V., Kosaka, P. \& Calleja, M. Quantification of the surface stress in microcantilever biosensors: revisiting Stoney's equation. Nanotechnology 23, 475702 (2012).

28. Hertz, H. Über die Berührung fester elasticher Körper. J. Reine Angew. Math. 92, 156-171 (1881)

29. Chaudhury, M. K., Weaver, T., Hui, C. \& Kramer, E. Adhesive contact of cylindrical lens and a flat sheet. J. Appl. Phys. 80, 30-37 (1996).

30. Barquins, M. Adherence and rolling kinetics of a rigid cylinder in contact with a natural rubber surface. J. Adhesion 26, 1-12 (1988).

31. Gaiduk, A., Yorulmaz, M., Ruijgrok, P. \& Orrit, M. Room-temperature detection of a single molecule's absorption by photothermal contrast. Science 330, 353-356 (2010).

32. Celebrano, M., Kukura, P., Renn, A. \& Sandoghdar, V. Single-molecule imaging by optical absorption. Nat. Photonics 5, 95-98 (2011).

\section{Acknowledgments}

We acknowledge financial support from the Spanish Science Ministry (MINECO) through projects MAT2012-36197 and from European Research Council through Starting Grant NANOFORCELLS (ERC-StG-2011-278860). 


\section{Author contributions}

All the authors conceived and designed the work. J.J.R. and V.P. carried out modeling and simulations. J.J.R. and J.T. developed the theoretical model. J.T., P.K. and M.C. wrote this manuscript with inputs from all authors. All authors analyzed the data, discussed the results and commented on the manuscript.

\section{Additional information}

Supplementary information accompanies this paper at http://www.nature.com/ scientificreports

Competing financial interests: The authors declare no competing financial interests.
How to cite this article: Ruz, J.J., Tamayo, J., Pini, V., Kosaka, P.M. \& Calleja, M. Physics of Nanomechanical Spectrometry of Viruses. Sci. Rep. 4, 6051; DOI:10.1038/srep06051 (2014).

(c) (i) $\Theta$ This work is licensed under a Creative Commons Attribution-NonCommercialNoDerivs 4.0 International License. The images or other third party material in this article are included in the article's Creative Commons license, unless indicated otherwise in the credit line; if the material is not included under the Creative Commons license, users will need to obtain permission from the license holder in order to reproduce the material. To view a copy of this license, visit http:// creativecommons.org/licenses/by-nc-nd/4.0/ 